\title{
A Theoretical Note on the Optimal Dependability and Speed of Container Inspections in Invasive Species Management ${ }^{1}$
}

\author{
Amitrajeet A. BATABYAL ${ }^{2}$
}

\begin{abstract}
The extant literature tells us that a seaport inspector who places a large (small) weight on biological invasion damage control relative to economic cost reduction will, ceteris paribus, inspect ships with containers more (less) stringently. Therefore, in this theoretical note we analyze the decision problem faced by a seaport inspector who places equal weight on the above two objectives. Specifically, we first present a stylized model of biological invasions. Next, we derive an expression for the long run expected net cost (LRENC) of inspections. Finally, we show that the optimal dependability and speed of inspections is the solution to this LRENC minimization problem.
\end{abstract}

JEL Classification: Q580, L510

Keywords: Alien Species, Dependability, Inspections, Speed, Uncertainty

\section{Introduction}

It is now well understood that airplanes, ships, and trucks have transported and continue to transport alien plant and animal species (also known as invasive and non-native species) from one region of the world to another. As far as airplanes are concerned, alien species have occasionally managed to lodge themselves in the landing gear and, by doing so, they have traveled as stowaways from one region of the world to another. Similarly, many marine alien species have been introduced unintentionally into a region by ships dumping their ballast water. Cargo ships routinely carry ballast water in order to augment vessel stability when they are not carrying full loads. When these ships come into a seaport, this ballast water must be released before cargo can be loaded. This means of species introductions is noteworthy and very recently the question of managing alien species that have been introduced into a particular region by means of the dumping of ballast water has attracted the attention of researchers. ${ }^{3}$

As far as ships and trucks are concerned, containers-routinely used to transport cargo from one region to another-are the primary means by which alien species have been unintentionally introduced into a particular region. In this regard, it is vital to comprehend that alien species can remain undetected in containers for extended periods of time. Further, the material-such as wood - that is often used to pack the cargo in the containers may itself contain alien species. In the case

${ }^{1}$ Batabyal thanks Yoshiro Higano and two anonymous referees for their helpful comments on previous versions of this theoretical note. In addition, he acknowledges financial support from the USDA's PREISM program by means of Cooperative Agreement 43-3AEM-4-80100 and from the Gosnell endowment at RIT. The usual disclaimer applies.

${ }^{2}$ Department of Economics, Rochester Institute of Technology, 92 Lomb Memorial Drive, Rochester, NY 14623-5604, USA. Internet aabgsh@ rit.edu

${ }^{3}$ See Yang and Perakis (2004), Batabyal et al. (2005), and Batabyal and Beladi (2006) for more on this literature.

Received: 31 October 2006 Accepted : 5 August 2007

(C) 日本地域学会 (JSRSAI) 2008 
of maritime shipments, a joint report from the United States Department of Agriculture (USDA), the Animal and Plant Health Inspection Service (APHIS), and the United States Forest Service (USFS) has remarked that roughly $51.8 \%$ of such shipments contain solid wood packing materials and that infection rates for solid wood packing materials are significant (USDA, APHIS, and USFS (2000, p. 25)). As an example, inspections of wooden spools from China uncovered infection rates between $22 \%$ and $24 \%$ and inspections of braces for granite blocks imported into Canada were found to contain live insects $32 \%$ of the time (USDA, APHIS, and USFS (2000, pp. 27-28)).

The invasions caused by the alien species that we have been discussing generally thus far have frequently turned out to be very costly for society. For the United States alone, the dollar value of these costs is enormous. In this regard, the Office of Technology Assessment (OTA (1993)) has calculated that the Russian wheat aphid caused $\$ 600$ million worth of crop damage between 1987 and 1989. Similarly, Pimentel et al. (2000) have computed the total costs of all alien species at around $\$ 137$ billion per year. In addition to these economic costs, alien species have given rise to serious biological damage as well. For instance, Vitousek et al. (1996) have shown that alien species can modify ecosystem processes, act as vectors of diseases, and diminish biological diversity. Further, Cox (1993) has observed that out of 256 vertebrate extinctions with a known cause, 109 are the outcome of biological invasions. The implication of the discussion in this paragraph is clear : Alien species have frequently been a great menace to society.

There are two kinds of actions - pre-invasion and post-invasion - that a manager or an inspector, interested in precluding biological invasions, can take. The objective of this theoretical note is to shed light on the attributes of one kind of pre-invasion action, namely, inspections. Therefore, we now briefly review four papers on inspections that are relevant to the analysis that is undertaken here. ${ }^{4}$ McAusland and Costello (2004) show that when one takes a dynamic view and considers the future impacts of current species introductions, one is naturally led to favor more stringent inspections. Batabyal et al. (2005) have analyzed $M / M / 1$ and $M / M / 2$ queuing models of inspections and have concluded that there is a tradeoff between economic cost minimization and inspection stringency in alien species management. Batabyal and Lee (2006) have analyzed container inspection policies in three scenarios. Their analysis shows that the long run expected net cost (LRENC) of inspections when inspection times are either deterministic or probabilistic is typically higher than the corresponding LRENC when inspection times are negligible. Finally, DeAngelo et al. (2007) have analyzed this tradeoff question with the more general $M / G / 1$ queuing model. Their basic result is that there may be, but there does not have to be, a tradeoff between economic cost minimization and inspection stringency in alien species management.

In the context of the Batabyal et al. (2005) and the DeAngelo et al. (2007) papers, greater (lesser) inspection stringency reflects a heightened (decreased) concern for the potential damage from one or more biological invasions. Therefore, a seaport inspector who places a large (small) weight on biological invasion damage control relative to economic cost reduction will, ceteris paribus, inspect ships with containers more (less) stringently. These findings raise the following important question: How should inspections be conducted by a seaport inspector who places equal weight on the above two objectives? The reader should understand that such an "equal weight" placing individual will be interested not only in the dependability of inspections but also on the speed with which these inspections are conducted. This is because the dependability of inspections proxies the seaport inspector's concern for biological invasion damage control. In addition, the speed of inspections proxies this seaport inspector's concern for economic cost reduction.

Despite the salience of the above question, to the best of our knowledge, the extant literature has not analyzed the question of determining the optimal dependability and the optimal speed of

${ }^{4}$ For more on the literature on post-invasion actions, see Eiswerth and Johnson (2002), Finoff and Tschirhart (2005), and Gutierrez and Regev (2005). 
container inspections in alien species management. Given this state of affairs, in our theoretical note, we analyze the decision problem faced by a seaport inspector who places equal weight on the above two objectives. To this end, we first present a stylized model of biological invasions in an arbitrary national seaport. Next, we use the theory of renewal-reward processes ${ }^{5}$ and derive an expression for the LRENC of inspections at this seaport. Finally, we show that the optimal dependability and speed of inspections is the solution to this LRENC minimization problem. In this regard, the reader should note that the LRENC is an objective function that our seaport inspector optimizes. It is not a method for addressing efficiency. In addition, it is not the case that our seaport inspector is concerned only about efficiency. In fact, as explained in the previous paragraph, this seaport inspector is concerned about equity in the sense that he places equal weight on the twin issues of biological invasion damage control and economic cost reduction.

The rest of this theoretical note is organized as follows. Section 2 describes a stylized model of biological invasions and the container inspection dependability and speed choice problem faced by a representative inspector at a national seaport. Section 3 first derives an expression for the LRENC of inspections and then this section shows that the optimal dependability and speed that is sought by our representative inspector can be obtained by minimizing the above mentioned LRENC with respect to an appropriately specified decision variable. Section 4 concludes and offers suggestions for future research on the subject of alien species management. Before we proceed to the next two substantive sections, we wish to reiterate unequivocally that this contribution of ours ought to be viewed as a theoretical note. In other words, a key objective of this contribution is to provide a conceptual analysis of the speed and dependability issue that we have talked about thus far. Our objective here is not to analyze collected data and nor is it to provide an exhaustive numerical analysis of the speed and dependability issue. Although we hope that seaport authorities and individuals in government departments such as customs will find our theoretical note interesting, in the final analysis, this theoretical note is directed primarily, but not exclusively, at the academic research community.

\section{A Stylized Model of Biological Invasions}

Consider an arbitrary national point of entry such as a seaport. Ships arrive at this seaport in accordance with a stationary Poisson process with rate $\lambda>0$. Upon arrival, ships typically unload their containers carrying cargo. The arrival of these ships with containers coincides with the arrival of a whole host of potentially dangerous biological organisms. Now, before this incoming cargo can be moved to various points in the interior of the country under consideration, the ships with containers must first be inspected. Obviously, the purpose of this preventive activity is to decrease the likelihood of the occurrence of one or more biological invasions. We assume that the arrival rate of the various potentially deleterious biological organisms is proportional to the arrival rate of the ships at our seaport. Therefore, we shall not model the possibly injurious biological organisms directly. Instead, we shall focus on the ships that bring these organisms to our seaport.

Our representative inspector would like to prevent invasions by the potentially deleterious biological organisms. However, as discussed in section 1, this inspector places equal weight on the objective of economic cost reduction and on the objective of biological invasion damage control. Therefore, this inspector will not want to take an inordinately long amount of time to conduct meaningful inspections. Now, suppose that our representative inspector can employ one of two possible procedures to inspect arriving ships. When this inspector employs the standard-but not very dependable-procedure, the random amount of time needed to inspect a particular ship and its containers is distributed in accordance with a particular probability distribution function with mean

\footnotetext{
${ }^{5}$ See Ross (1996, pp. 132-140) and Tijms (2003, pp. 39-50) for more on renewal-reward processes.
} 
$\mu>0$ and standard deviation $\sigma>0$.

When the standard procedure is employed, the inspector incurs costs and obtains benefits from two sources. The first source of net cost, the difference between total cost and total benefit, arises from things like the expense of operating the relevant equipment and holding the containers in a secure area (a cost) and from the positive impact on society that results from diminishing the likelihood of one or more biological invasions (a benefit). We model this first source of net cost by supposing that our inspector incurs net cost at the rate of $c>0$ dollars for each time unit that the standard procedure is employed. The second source of net cost arises from things like the negative impact on society from the slowdown in economic activity while ships with containers are being inspected (a cost) and from the determination of whether the ship containers actually contain what they are supposed to contain (a benefit). We account for this second source of net cost by supposing that our inspector incurs net cost at the rate of $h>0$ dollars per ship in the seaport.

We have already noted that our representative inspector places equal weight on biological invasion damage control and on the economic cost imposed on society by inordinately lengthy inspections. Therefore, this individual will want an inspection procedure that is both fast and dependable. Recall that the dependability of the standard inspection procedure is an issue. Therefore, given the discussion in the previous paragraph, we suppose that it is also possible for our inspector to eschew the standard procedure and, instead, use an alternate procedure with dependability and speed denoted by $r$. As one might expect, this higher level of both dependability and speed can only be achieved by incurring additional costs. We model this feature of the problem by supposing that the first source of net cost when our inspector uses this alternate-and, relative to the standard procedure, $r$ times faster and more dependable-procedure is quadratic and equal to $\mathrm{Cr}^{2}$ per unit time. The second source of net cost stays unchanged. Finally, we assume that our inspector attends to one ship and its containers at a time and that there is no shortage of docks for arriving ships in the seaport. In this theoretical note, we are interested primarily in the optimal dependability and speed of container inspections. Hence, the task before us now is to derive an expression for the LRENC of inspections as a function of the dependability and speed level $r$.

\section{The LRENC of Inspections}

To derive the relevant expression for the $L R E N C$ of inspections, we shall use standard results for the $M / G / 1$ queuing model ${ }^{6}$ and the so called renewal-reward theorem. ${ }^{7}$ Using these known results for the $M / G / 1$ queuing model, we can tell that the long run proportion of time that an inspection procedure is in use is given by

$$
\rho(r)=\lambda r / \mu \text {. }
$$

Our next task is to compute the long run expected number of ships that are inspected in our seaport. To compute this expression, we use equation 8.34 in Ross (2003, p. 508) adapted to our problem. This procedure tells us that the expectation we seek is

$$
\rho(r)+\left\{\lambda^{2} / 2 r^{2}\right\}\left[\left(\mu^{2}+\sigma^{2}\right) /\{1-\rho(r)\}\right] .
$$

Given the expressions in (1) and (2), the renewal-reward theorem tells us that, with probability one, the $L R E N C$ of inspections per unit time is given by

$$
\text { LRENC }=(c+h)(\lambda r / \mu)+h\left(\lambda^{2} / 2 r^{2}\right)\left[\left(\mu^{2}+\sigma^{2}\right) /\{1-(\lambda r / \mu)\}\right] .
$$

${ }^{6}$ See Ross (2003, pp. 507-519) and Tijms (2003, pp. 345-360) for more on these standard results for the $M / G / 1$ queuing model.

${ }^{7}$ Given a renewal-reward stochastic process, the renewal-reward theorem tells us that the long run expected reward per unit time for this process is equal, with probability one, to the expected reward earned in one cycle divided by the expected time it takes to complete this cycle. For more on this theorem, see Ross (1996, p. 133) and Tijms (2003, p. 41). 
Having computed the LRENC of inspections, we are now in a position to state our representative inspector's optimization problem. Specifically, this inspector chooses the container inspection dependability and speed level $r$ to minimize the LRENC from inspections. Formally, our representative inspector solves

$$
\min _{\{r\}}\left[(c+h)(\lambda r / \mu)+h\left(\lambda^{2} / 2 r^{2}\right)\left[\left(\mu^{2}+\sigma^{2}\right) /\{1-(\lambda r / \mu)\}\right]\right] .
$$

Treating $r$ as a continuous control variable and using calculus, we see that the optimal dependability and speed $r^{*}$ that minimizes the representative inspector's LRENC is given implicitly by the most reasonable solution to the following polynomial

$$
2 r^{3}(c+h)(\mu-\lambda r)=h \lambda \mu^{2}\left(\mu^{2}+\sigma^{2}\right)(2 \mu-3 \lambda r) .
$$

From an analytical perspective, this is about as far as we can go in determining the properties of the optimal inspection dependability and speed $r^{*}$. To obtain additional insights into the properties of the optimal $r^{*}$ there are two ways to proceed. One could either collect actual data on the stochastic parameters $(\lambda, \mu, \sigma)$ and the cost parameters $(c, h)$ of the model and then analyze the behavior of the polynomial in (5). Alternately, one could also use artificial data along with numerical methods to study the properties of the polynomial in (5).

\section{Conclusions}

In this theoretical note, we studied the decision problem faced by a seaport inspector who places equal weight on the seemingly competing objectives of economic cost reduction and biological invasion damage control. We argued that such an inspector values both dependability and speed in the conduct of container inspections. Therefore, we first presented a stylized model of biological invasions in an arbitrary national point of entry such as a seaport. Next, we derived an expression for the LRENC of container inspections. Finally, we showed that the optimal dependability and speed of container inspections is given by the solution to a specific LRENC minimization problem.

Our analysis here can be extended in a number of directions. In what follows, we make two suggestions for extending the research described in this theoretical note. First, it would be useful to analyze a scenario in which point of origin information for the arriving ships is used to first place ships in dissimilar inspection classes. Only then are container inspections of the sort described in this theoretical note used to reduce the likelihood of one or more biological invasions. Second, it would also be helpful to determine whether the results we have obtained in this paper with the Poisson process carry over to more general renewal processes. Studies of inspections in alien species management that incorporate these aspects of the problem into the analysis will provide additional insights into a regulatory task that has considerable economic and biological implications.

\section{References}

[1] Batabyal, A.A., Beladi, H. and Koo, W.W., "Maritime Trade, Biological Invasions, and the Properties of Alternate Inspection Regimes," Stochastic Environmental Research and Risk Assessment, Vol. 19, 2005, pp. 184-190.

[2] Batabyal, A.A. and Beladi, H., "International Trade and Biological Invasions: A Queuing Theoretic Analysis of the Prevention Problem," European Journal of Operational Research, Vol. 170, 2006, pp. 758-770.

[ 3 ] Batabyal, A.A. and Lee, D.M., "The Infinitesimal, the Deterministic, and the Probabilistic: Alternate Container Inspection Policies in Invasive Species Management," Biological Invasions, Vol. 8, 2006, pp. 1663-1671.

[ 4 ] Cox, G.W., Conservation Ecology, W.C. Brown Publishers, Dubuque, Iowa, 1993.

[5] DeAngelo, G., Batabyal, A.A. and Kumar, S., On Economic Cost Minimization Versus Biological 
Invasion Damage Control, in A.G.J.M. Oude Lansink, (Ed.), New Approaches to the Economics of Plant Health, Springer-Verlag, Heidelberg, Germany, 2007.

[6 ] Eiswerth, M.E. and Johnson, W.S., "Managing Nonindigenous Invasive Species: Insights from Dynamic Analysis,” Environmental and Resource Economics, Vol. 23, 2002, pp. 319-342.

[ 7 ] Finoff, D. and Tschirhart, J., "Identifying, Preventing, and Controlling Invasive Plant Species Using Their Physiological Traits,” Ecological Economics, Vol. 52, 2005, pp. 397-416.

[8] Gutierrez, A.P. and Regev, U., "The Bioeconomics of Tritrophic Systems: Application to Invasive Species,” Ecological Economics, Vol. 52, 2005, pp. 383-396.

[ 9 ] McAusland, C. and Costello, C., "Avoiding Invasives: Trade-Related Policies for Controlling Unintentional Exotic Species Introductions,” Journal of Environmental Economics and Management, Vol. 48, 2004, pp. 954-977.

[10] Office of Technology Assessment (OTA), Harmful Non-Indigenous Species in the United States, OTA-F-565, Washington, District of Columbia, 1993.

[11] Pimentel, D., Lach, L., Zuniga, R. and Morrison, D., "Environmental and Economic Costs of Nonindigenous Species in the United States,” BioScience, Vol. 50, 2000, pp. 53-65.

[12] Ross, S.M., Stochastic Processes, $2^{\text {nd }}$ edition. Wiley, New York, New York, 1996.

[13] Ross, S.M., Introduction to Probability Models, $8^{\text {th }}$ edition. Academic Press, Amsterdam, The Netherlands, 2003.

[14] Tijms, H.C., A First Course in Stochastic Models, Wiley, Chichester, UK, 2003.

[15] USDA, APHIS, and USFS, Pest Risk Assessment for Importation of Solid Wood Packing Materials into the United States. http://www.aphis.usda.gov/ppq/pra/swpm/complete.pdf, 2000 .

[16] Vitousek, P.M., D’Antonio, C.M., Loope, L.L. and Westbrooks, R., "Biological Invasions as Global Environmental Change,” American Scientist, Vol. 84, 1996, pp. 468-478.

[17] Yang, Z. and Perakis, A.N., "Multiattribute Decision Analysis of Mandatory Ballast Water Treatment Measures in the US Great Lakes," Transportation Research Part D, Vol. 9, 2004, pp. 81-86. 